\title{
Photoconductivity and dielectric studies of potassium pentaborate crystal (KB5)
}

\author{
V JOSEPH*, S GUNASEKARAN ${ }^{\dagger}$ and V SANTHANAM ${ }^{\ddagger}$ \\ Department of Physics, Loyola College, Chennai 600 034, India \\ Department of Physics, Pachaiyappa's College, Chennai 600 030, India \\ *Department of Physics, Presidency College, Chennai 600 005, India
}

MS received 30 October 2002; revised 27 February 2003

\begin{abstract}
Single crystal of potassium pentaborate (KB5) has been grown by solution growth technique. FTIR and laser Raman measurements are carried out to make a qualitative analysis on KB5 crystal. Dielectric behaviour of the KB5 crystal has been studied in the microwave region using $\mathrm{K}$-band microwave bench equipped with the Gunn Oscillator guided with rectangular wave-guide. To confirm the suitability of this crystal as electro optic device, its dielectric behaviour with the change of frequency has also been investigated. Photoconductivity studies were also carried out on this material. It was interesting to observe that the KB5 crystal exhibited negative photoconductivity.
\end{abstract}

Keywords. Photoconductivity; dielectric constant; dark current.

\section{Introduction}

The fast development in the field of optoelectronics has stimulated the search for highly new non-linear optical crystals for efficient signal processing. New non-linear optical (NLO) frequency conversion materials can have a significant impact on laser technology (Belt et al 1985), optical communication (Clark 1988) and optical data storage technology (Gambino 1990). Materials with nonlinear electro optic properties have a role in modern optoelectronics that is analogous to that of non-linear electronic circuit elements in conventional electronics. Potassium pentaborate (KB5) is one such efficient nonlinear material which is used for second harmonic generation. The experiments conducted by Becker (1998) on NLO materials proved that borate compound materials are superior to other commonly used materials for UV applications. KB5 is a desirable NLO material which exhibits a low angular sensitivity and hence, proved useful for type II second harmonic generation (SHG) (Voitsekhovskii et al 1982). KB5 is orthorhombic with four molecules in the unit cell of dimensions $a=11.062 \AA, b=11.175 \AA, c=$ 9.041 $\AA$. The dielectric properties of KB5 was not reported in the microwave region and the vibrational assignments were incomplete. In the present work, attention has been given to characterize this crystalline material through FTIR and laser Raman spectral measurements along with dielectric and photoconductivity studies.

\footnotetext{
*Author for correspondence
}

\section{Experimental}

The starting material was synthesized by stoichiometric incorporation of potassium carbonate (AR grade) and boric acid (AR grade) taken in the appropriate ratio (Ramachandra Raja et al 1993). The calculated amount of salts was dissolved in deionized water at room temperature. KB5 salt was synthesized according to the reaction,

$$
\begin{aligned}
\mathrm{K}_{2} \mathrm{CO}_{3}+10 \mathrm{H}_{3} \mathrm{BO}_{3} \rightarrow 2\left[\mathrm{~K}\left(\mathrm{H}_{4} \mathrm{~B}_{5} \mathrm{O}_{10}\right) \cdot 2 \mathrm{H}_{2} \mathrm{O}\right] & +7 \mathrm{H}_{2} \mathrm{O} \\
& +\mathrm{CO}_{2} \uparrow
\end{aligned}
$$

The purity of the synthesized salt was further increased by successive recrystallization process. Single crystals of KB5 are grown by slow and controlled evaporation of the solvent using a constant temperature bath. The supersaturated solution was kept in a constant temperature bath at $305 \mathrm{~K}$ in a polyethylene container with stirrer made of the same material. Crystals prepared by spontaneous nucleation were used as seed crystals. The period of growth ranged from 60 to 90 days.

\section{Spectral analysis}

The FTIR spectrum of KB5 was recorded on BRUKER IFS 66v Model FTIR spectrophotometer using KBr pellet. The laser Raman spectrum of KB5 was recorded on DILOR-Z24 Spectrophotometer. The spectra are presented in figures 1 and 2, and the observed frequencies with their assignments are summarized in table 1 .

The $\mathrm{B}-\mathrm{O}$ vibrations of borate crystals have their absorption bands in the frequency region $784-1438 \mathrm{~cm}^{-1}$ (Ross 1969; Clothup 1975; Chryssikos 1991; Chryssikos et al 
1991). The strong bands observed in the IR spectrum of KB5 at $784 \mathrm{~cm}^{-1}$ and $923 \mathrm{~cm}^{-1}$ have been assigned to ring $\mathrm{B}-\mathrm{O}$ symmetric stretching vibrations. In the laser Raman spectrum these bands appear at $788 \mathrm{~cm}^{-1}$ and $918 \mathrm{~cm}^{-1}$, respectively. The ring $\mathrm{B}-\mathrm{O}$ asymmetric stretching vibrations appear at $1103 \mathrm{~cm}^{-1}, 1251 \mathrm{~cm}^{-1}$ and $1356 \mathrm{~cm}^{-1}$ with very strong intensity. The very strong peak at $1027 \mathrm{~cm}^{-1}$ in the infrared spectrum has been attributed to $\mathrm{B}-\mathrm{O}$ terminal symmetric stretching vibration. The $\mathrm{B}-\mathrm{O}$ terminal asymmetric stretching is observed in the IR spectrum at $1438 \mathrm{~cm}^{-1}$ with strong intensity. The very strong band observed at $3445 \mathrm{~cm}^{-1}$ has been assigned to $\mathrm{OH}$ stretching vibration.

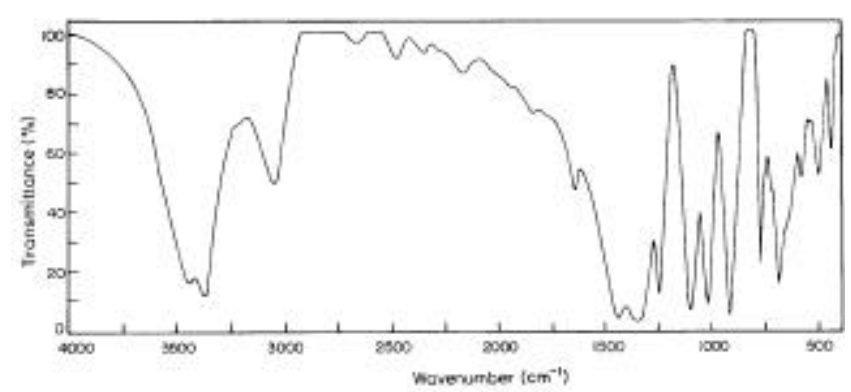

Figure 1. FT-IR spectrum of KB5.

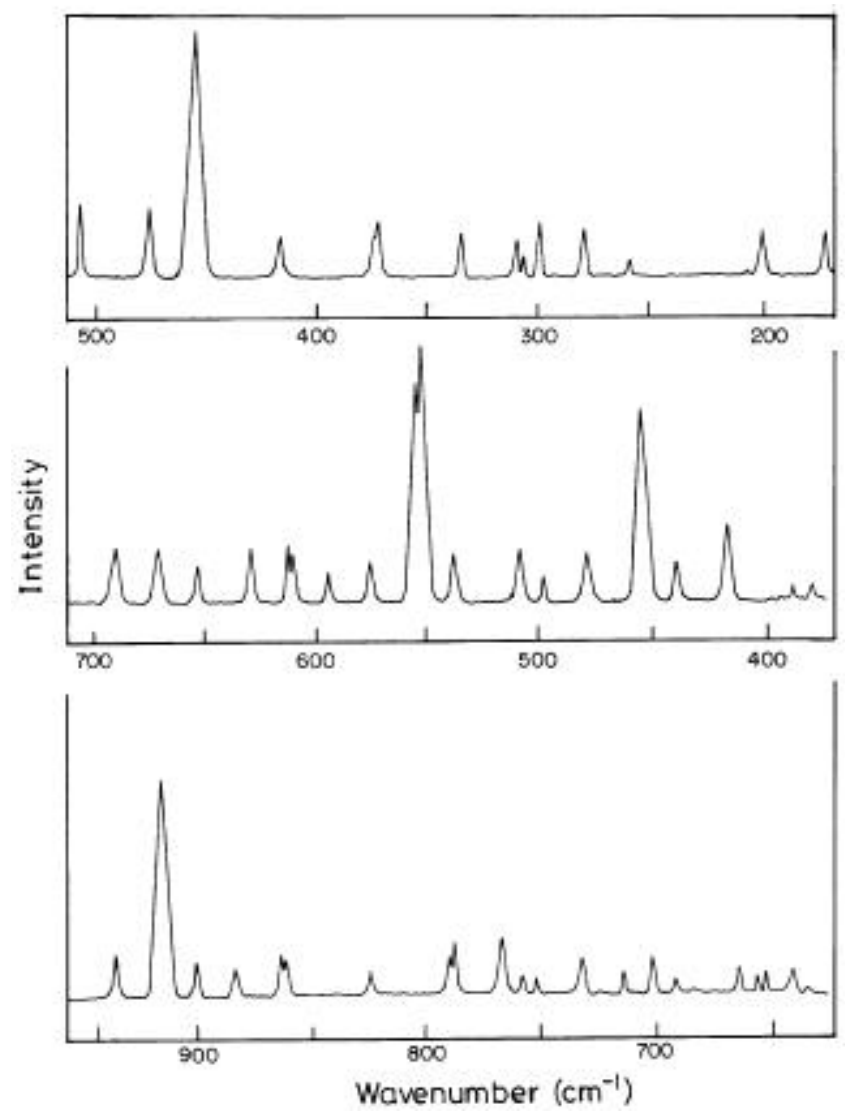

Figure 2. Laser Raman spectrum of KB5.
The bands observed in the Raman spectrum at $456 \mathrm{~cm}^{-1}$ and $504 \mathrm{~cm}^{-1}$ are assigned to ring OBO symmetric bendings. The corresponding frequencies in the IR spectrum are observed at $454 \mathrm{~cm}^{-1}$ and $509 \mathrm{~cm}^{-1}$. The ring asymmetric bending vibrations are found at $655 \mathrm{~cm}^{-1}$ and $695 \mathrm{~cm}^{-1}$ with strong intensity. The medium intensity bands appearing at $552 \mathrm{~cm}^{-1}$ and $591 \mathrm{~cm}^{-1}$ are attributed to terminal ring OBO bendings. The terminal asymmetric bending vibration appears at $728 \mathrm{~cm}^{-1}$ and corresponding vibration in the Raman spectrum appears at $731 \mathrm{~cm}^{-1}$.

\section{Dielectric measurements}

The K-band microwave test bench equipped with Gunn Oscillator was used to measure the dielectric constant of the sample. The dimension of the wave-guide used in the present case is $1.1 \times 0.4 \mathrm{~cm}$ and hence the crystals are accordingly shaped to this size. Standing wave measurements have been carried out to determine the dielectric constants of the KB5 crystal. The functional block diagram of a microwave bench is shown in figure 3 .

A direct and accurate method of Robert and VonHipple was used to measure the dielectric constant of the sample (Roberts and Von-Hipple 1956; Sisodia and Raghuvanshi 1990). The Von-Hipple method is based on the measurement of shift in the minimum of standing wave produced by a short circuit when a sample of

Table 1. FTIR and laser Raman frequencies of KB5 crystal and their assignments.

\begin{tabular}{lcl}
\hline FTIR $\left(\mathrm{cm}^{-1}\right)$ & Laser Raman $\left(\mathrm{cm}^{-1}\right)$ & \multicolumn{1}{c}{ Assignment } \\
\hline & 373 & OBO deformation \\
454 & 456 & OBO ring bending \\
509 & 509 & OBO ring bending \\
552 & 555 & OBO terminal bending \\
591 & - & OBO terminal bending \\
655 & - & OBO ring asym. bending \\
695 & - & OBO ring asym. bending \\
728 & 731 & OBO ter. asym. bending \\
784 & 788 & B-O ring stretching \\
923 & 918 & B-O ring stretching \\
1027 & - & B-O ter. stretching \\
1103 & - & B-O asym. stretching \\
1251 & - & B-O asym. stretching \\
1356 & - & B-O asym. stretching \\
1438 & - & B-O ter. asym. stretching \\
3445 & - & OH stretching \\
\hline
\end{tabular}

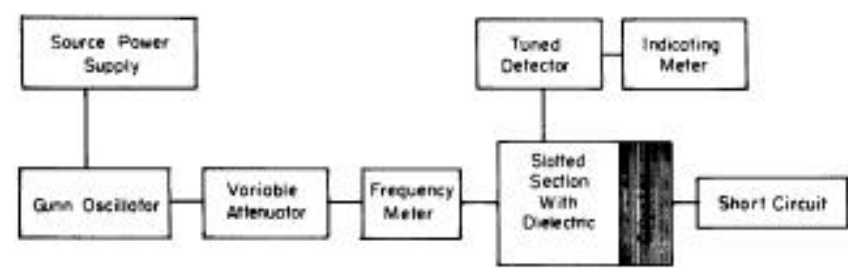

Figure 3. Functional block diagram of a microwave bench. 
dielectric is placed in front of the short circuit. The shift, $\Delta$, in the minimum of standing wave was caused due to the presence of the sample. To get common solution, measurements were carried out on the material with four different faces.

The dielectric constant $\left(\varepsilon_{\mathrm{r}}\right)$ of the crystals is evaluated by using

$$
\beta_{\mathrm{d}}=\frac{2 \pi}{\lambda_{0}}\left[\varepsilon_{\mathrm{r}} \mu_{\mathrm{r}}-\left(\frac{\lambda_{0}}{2 a}\right)^{2}\right]^{\frac{1}{2}},
$$

where $\beta_{\mathrm{d}}$ is the phase constant of the wave in the dielectric medium, $\mu_{\mathrm{r}}$ the relative permeability of the material taken as unity for high frequency and $\lambda_{0}$ the free space wavelength and $a$ the wider dimension of the wave guide.

The dielectric constants of the KB5 crystal at different frequencies are listed in table 2.

\section{Photoconductivity studies}

The KB5 crystal was fixed on a glass plate and silver contacts were made over the sample to ensure good ele-

Table 2. Dielectric constants of KB5 crystal at different frequencies.

\begin{tabular}{lc}
\hline Frequency $(\mathrm{GHz})$ & Dielectric constant $\left(\varepsilon_{\mathrm{r}}\right)$ \\
\hline $19 \cdot 6$ & 3.9589 \\
& $3 \cdot 8440$ \\
4.0185 \\
& 3.9590 \\
$22 \cdot 7$ & $3 \cdot 8756$ \\
& $3 \cdot 7450$ \\
& 3.9185 \\
& 3.8560 \\
\hline
\end{tabular}

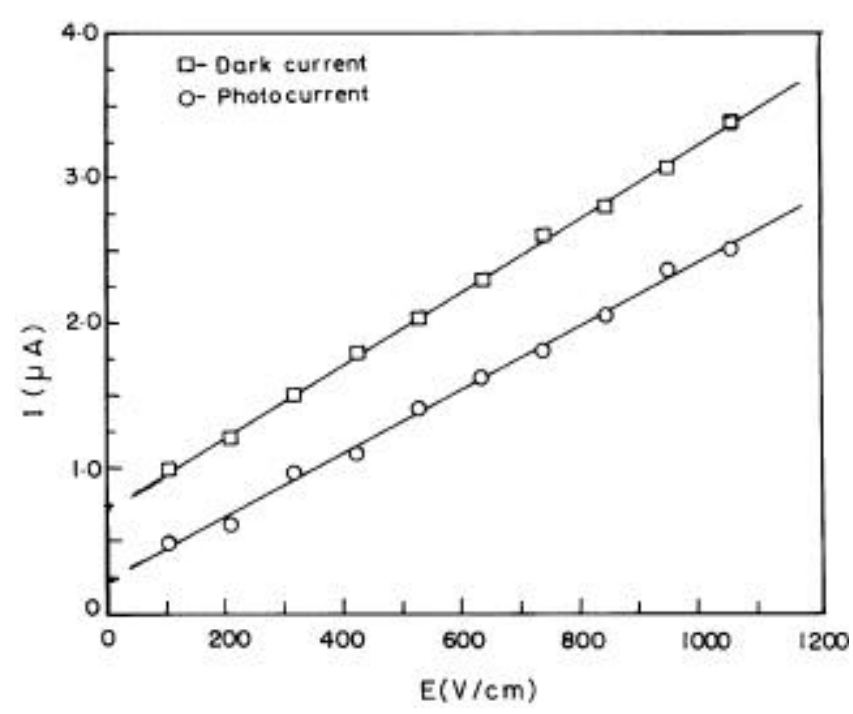

Figure 4. Plot of photocurrent/dark current vs applied electric field for KB5. ctrical contacts. The sample was connected in series to a d.c. power supply and a picoammeter (Keithley 480). The applied electric field to the sample was varied from 100 to $1100 \mathrm{~V} \mathrm{~cm}^{-1}$ without exposing the sample to any radiation and the corresponding dark current was recorded. The material was then exposed to radiation from a halogen lamp and the photocurrent was measured for various electric fields. Figure 4 shows the plot of dark current and photocurrent against the electric field. It is interesting to note that the photocurrent is less than the dark current, which is termed as negative photoconductivity.

The phenomenon of negative photoconductivity is explained successfully by Stockmann model (Joshi 1990). The negative photoconductivity in a solid is due to the reduction in the number of charge carriers or their life time, in the presence of radiation (Bube 1960). According to this model, the forbidden gap in the material contains two energy levels in which one is situated between the Fermi level and the conduction band while the other is located close to the valence band. The second state has high capture cross sections for electrons and holes. As it captures electrons from the conduction band and holes from the valence band, the number of charge carriers in the conduction band gets reduced and the current decreases in the presence of radiation.

\section{Conclusion}

A satisfactory vibrational band assignment has been made available for KB5 crystal through FTIR and laser Raman spectroscopy. Dielectric behaviour of the KB5 crystal has also been investigated in the K-band microwave region. Further, the conductivity of the crystal was studied under dark and illuminated conditions and it was found to exhibit negative photoconductivity.

\section{Acknowledgement}

One of the authors (VJ) is thankful to the University Grants Commission, Government of India, for the award of a teacher fellowship.

\section{References}

Becker P 1998 Adv. Mater. 10979

Belt R F, Gashurov G and Liu Y S 1985 Laser Focus 10110

Bube R H 1960 Photoconductivity of solids (New York: Wiley)

Chryssikos G D 1991 J. Raman Spectrosc. 22645

Chryssikos G D, Kapoutsis J A, Patsis A P and Kamitsos E I 1991 Spectrochim. Acta 471117

Clark R S 1988 Photonics Spectra 22135

Clothup N B 1975 Introduction to infrared and Raman spectroscopy (London: Academic Press) II ed. 
Gambino R J 1990 Bull. Mater. Res. Soc. 1520

Joshi N V 1990 Photoconductivity (New York: Marcel Dekker)

Ramachandra Raja C, Gobinathan R and Gnanam F D 1993

Cryst. Res. Technol. 28453

Ross S D 1969 J. Mol. Spectrosc. 29131
Roberts S and Von Hipple A 1956 J. Appl. Phys. 717

Sisodia M L and Raghuvanshi G S 1990 Basic microwave techniques (New Delhi: Wiley Eastern Ltd)

Voitsekhovskii V N, Nikolaevu V P and Velichko I A 1982 Sov. Phys. Crystallogr. 27322 